\title{
Estados y agentividad
}

\section{States and Agentivity}

JuAn Moreno Burgos [Juan.Moreno-Burgos@zsk.uni-regensburg.de]

Universität Regensburg/ HAW Landshut, Alemania

\begin{abstract}
RESUMEN
La agentividad es una noción lingüística que no puede ser abordada desde una única perspectiva. En la bibliografía se habla de 'control' o 'volición', lo cual remite a la estructura argumental de ciertos predicados. Sin embargo, aún no se ha llegado al fondo de la cuestión, ya que es necesario investigar hasta qué punto se trata de un significado primario. El presente artículo explica que las lecturas agentivas llegan por vía inferencial y modal. Esto es, se trataría de un valor semántico derivado que solo surge con determinadas clases aspectuales: con eventos télicos y con estados, respectivamente. En este último caso, el factor inductor de la modalidad será identificado con la perfectividad o las formas de imperativo.
\end{abstract}

\section{Palabras clave}

modalidad; telicidad; agente; causa; verbo pronominal; estatividad

\begin{abstract}
Agentivity is a linguistic notion that cannot be described from only one perspective. Scholars usually account for it in terms of 'control' or 'volition', which refers to the argument structure of certain predicates. Nevertheless, it seems that the whole description of it remains incomplete, for it should be detailed whether this feature constitutes a primary meaning. The current paper explains that agentive interpretations arise by means of inferential or modal mechanisms. In other words, it constitutes a derived semantic value that emerges from specific aspectual classes: telic events and states. In this latter case, perfectivity or imperative forms will be defined as modality triggers.
\end{abstract}

KEYWORDS

modality; telicity; agent; cause; pronominal verb; stativity

RECIBIDO 2016-11-20; ACEPTADO 2017-02-09

Agradezco las observaciones de dos revisores anónimos por los comentarios realizados. 


\section{Introducción}

Según es referido en la gran mayoría de los estudios aspectuales, los predicados de una lengua se dividen en dos grandes clases: los estados y los eventos. A pesar de las múltiples explicaciones que se han ofrecido para distinguir entre ambos, existe un criterio que prima sobre el resto: los eventos expresan dinamicidad, pero no así los estados. No obstante, esta regla tan básica suele ser malinterpretada, debido probablemente a que Vendler (1957) empezó relacionando a los estados con la duratividad. Esta afirmación también se registra en trabajos más recientes como los de González Rodríguez (2011), Cuatero Otal y Horno Chéliz (2011) o Marín (2013); sin embargo, la duración implica un desarrollo temporal que solo puede ser asignable a los predicados dinámicos. Para zanjar esta cuestión, se hace indispensable reivindicar los trabajos de Beck (1987) y Moreno Cabrera (2003), quienes, desde diferentes enfoques, llegan a la conclusión de que los estados son atemporales; esto es, no-dinámicos. Solo partiendo de esta observación, a nuestro modo de ver definitiva, se puede abordar con garantías otros fenómenos lingüísticos relacionados. Veamos los siguientes ejemplos, tomados de Marín (2013) y Arche (2011):

(1) Juan fue cruel con su padre (Marín 2013: 63).

(2) Juan, sé $\{$ atento/ cruel/ amable\} (Arche 2011: 99).

Dichos autores explican las respectivas oraciones por el hecho de que el sujeto de la predicación se comporta de una manera determinada. ¿Cuál es, pues, el interés teórico por estos enunciados? Se trata de encajar las piezas de un puzle en el cual se espera que solo los eventos aparezcan vinculados a la noción de 'agentividad' y que, por tanto, esto debería excluir a los estados. Normalmente se aducen las pruebas sugeridas por Lakoff (1970) y Dowty (1979), a partir de las cuales se pretende demostrar que los estados no son compatibles con el imperativo ( ${ }^{\star}$ Sé español), con adverbios relacionados con la intencionalidad ( ${ }^{\star}$ Juan es español deliberadamente), con las oraciones finales ( ${ }^{*}$ Juan es español para fastidiar a su padre) o la perífrasis de Progresivo ( ${ }^{*}$ Juan está siendo español). El hecho de que existan contraejemplos como los de (1) y (2) se explica, según esta teoría, porque existiría una reinterpretación eventiva.

Ahora bien, admitiendo que existe una lectura agentiva evidente, debemos sin embargo interrogarnos sobre la naturaleza de dicha interpretación. Para ello estableceremos las siguientes bases:

- La agentividad no puede ser un criterio diferenciador entre estados y eventos, ya que se estarían confundiendo dos teorías independientes: por un lado, la teoría de las valencias de Tesnière (1959) y, por otro, la teoría aspectual de Vendler (1957).

- Desde la gramática de las valencias se considera que la noción de 'agente' constituye uno de los papeles temáticos en la estructura argumental de determinados verbos. Dicho papel remite a una entidad del mundo extralingüístico que es identificada con una persona.

- La existencia de un agente implica la correlación con un segundo elemento sobre el que este actúa (Juan abre la puerta) o en el que se posiciona (Juan ha ido a Jaén)․․

1 No nos detendremos en detallar una tipología completa de todos los argumentos que puedan existir, ni en cuestiones terminológicas relacionadas (tema, experimentante, objeto afectado, paciente, etc.). Tampoco ahondaremos en las diferencias entre estos y los complementos no-obligatorios o circunstantes. 
- En el caso de que la acción realizada conduzca de manera indirecta a un resultado (Juan ha derramado la leche), se le otorgará al sujeto el papel semántico de causa. Esto se aplica tanto a sujetos personales como no-personales ${ }^{2}$.

- Es necesario considerar a la agentividad y a la dinamicidad por separado, a pesar de que ambas nociones se puedan solapar en ciertos casos.

- La agentividad no siempre viene impuesta por la estructura argumental de los predicados, sino que esta puede llegar por otras vías.

A partir de estas bases, hemos estructurado nuestro trabajo en dos partes, en las cuales se examina, respectivamente, la relación de la agentividad con los eventos y con los estados. En el primer caso, mostramos por qué los eventos dinámicos no siempre son agentivos, ya que consideramos que la agentividad es un rasgo que solo debe ser aplicado a los predicados télicos. Dentro de esta lógica, explicamos que el pronombre que aparece acompañando a eventos como enamorarse sirve precisamente para cancelar dicha lectura agentiva. En el segundo caso, llegaremos al fondo de la problemática planteada en (1) y (2): la dinamización que se le atribuye a estas oraciones no llega a través de la adición de un supuesto papel temático de agente, sino que mostraremos que está relacionada con la modalidad; esto es, con el contenido de verdad de las preposiciones.

\section{Agentividad y eventos}

\subsection{La dinamicidad}

La agentividad es un fenómeno que debe ser puesto en relación con un cambio de estado; es decir, con la llamada telicidad. Esto se observa en predicaciones como Juan ha roto una carta, la cual implica un resultado del tipo "la carta está rota". Sin embargo, excluiremos predicaciones como Juan llamó a Ana por teléfono o Juan está mirando el cuadro, ya que aquí no se inaugura ningún estado de cosas. En nuestra opinión, se trata únicamente de situaciones dinámicas, pero no agentivas; esto es, son atélicas ${ }^{3}$. La noción de 'estado de cosas' debe entenderse aquí de una manera diferente a la de Dik (1997), ya que nosotros reservamos este término exclusivamente para los predicados no-dinámicos. La telicidad referida consiste, por tanto, en una transición entre dos estados.

En este sentido, queremos llamar la atención sobre el hecho de que la agentividad no constituye un primitivo, sino que es una propiedad derivada que llega por un mecanismo inferencial. Lo observamos a continuación:

2 Cf. Cano Cambronero (2013: 120). En este sentido podemos interrogarnos si estamos ante una causa o un agente cuando el sujeto es un animal: El perro rompió el muñeco frente a El viento rompió las ramas. Esta cuestión no será abordada aquí.

3 Vendler (1957) denomina actividades a los predicados atélicos. En una subdivisión más precisa, Bertinetto (1986) habla además de semelfactivos. En Moreno Burgos (2014a: 121-125) se indica que las segundas son idénticas a las primeras, con la salvedad de que a los semelfactivos se les superpone un componente pragmático acerca de su "corta" duración. 
(3) Me vuelvo a mirar. La mujer ha salido de su casa al oír el ruido, y desde la puerta [...], muy cerquita de mí, casi a mis espaldas, está llamando al perro (El País, 02/03/1980, CREA)4.

El predicado salir se caracteriza por conceptualizar la relación entre dos estados de cosas contrarios, los cuales pueden ser descritos como una transición entre no-estar-fuera y estarfuera. Así, partiendo del principio de temporalidad formulado por Moreno Cabrera (2003: 120123), el hecho de que una entidad se encuentre en un momento $t_{1}$ en un lugar determinado $y$ en un momento $t_{3}$ en otro diferente se interpreta forzosamente como que ha tenido lugar un desplazamiento autónomo ${ }^{5}$. En otras palabras, una entidad no puede estar en dos lugares al mismo tiempo (cf. Langacker 1987; Carlson 1978). Esta afirmación, que supone profundizar en la teoría de Pustejovsky (1991), implica defender que los eventos son predicados complejos que están formados por estados, los cuales son nucleares e indivisibles (Moreno Burgos 2014a). Veamos ahora esta otra oración:

(4) Abelardo, Stoichkov o Couto también verán el partido desde la grada. Óscar sigue en el más completo ostracismo, pero Roger y Celades sí han entrado en la lista ( $A B C$ electrónico, 31/08/1997, CREA).

Aquí tenemos igualmente un predicado télico que conceptualiza la transición entre no-estar y estar. Lo curioso es que en este caso el evento entrar no implica un desplazamiento físico, sino que se emplea en un sentido figurado: la inclusión en una lista. Puesto que, al contrario que en (3), no se vincula al sujeto con dos entidades locativas en un sentido propio, la lectura agentiva aparece aquí bloqueada.

Por otro lado, si los predicados dinámicos incluyeran en su semántica la agentividad, no se produciría la reinterpretación estativa que encontramos en ejemplos como los siguientes:

(5) La carretera va de Madrid a Zaragoza (Moreno Cabrera 2003: 123).

(6) El río baja desde la montaña a la costa (Moreno Cabrera 2003: 123).

Como vemos, los verbos que aparecen aquí (ir y bajar) remiten originalmente a eventos. Esto lo observamos más claramente si hacemos las transformaciones correspondientes: Juan va a Zaragoza, Juan baja a la costa. Sin embargo, la estatividad de (5) y (6), que remite a ciertas propiedades de los respectivos sujetos, se explica a partir de la cancelación del principio de temporalidad, ya que permite vincular a una entidad con dos lugares diferentes ${ }^{6}$. Nuestro razonamiento es simple: la ausencia de agentividad no siempre implica estatividad (p.e. enamorarse), mientras que una predicación no-dinámica ha de ser forzosamente estática.

Al diferenciar entre la agentividad por un lado y la dinamicidad por otro, podemos ofrecer la siguiente clasificación:

4 Los ejemplos que no remitan a los autores citados proceden del corpus CREA de la Real Academia Española (variedad geográfica de España).

5 El autor emplea la denominación Principio de la unidad espacio-temporal.

6 No consideramos, por tanto, que el proceso cognitivo que opera en la estativización de estos enunciados impliquen un movimiento figurado. Dicho movimiento figurado sí que está disponible en la oración (4). 


\begin{tabular}{|l|c|c|}
\cline { 2 - 3 } \multicolumn{1}{c|}{} & Agentividad & Dinamicidad \\
\hline Salir & Sí & Sí \\
\hline Enamorarse & No & Sí \\
\hline Escribir & No & Sí \\
\hline Escribir una carta & Sí & Sí \\
\hline
\end{tabular}

Figura 1. Agentividad y dinamicidad según este trabajo.

En este cuadro reflejamos que los eventos télicos pueden asociarse con la agentividad (salir) o estar disociados de ella (enamorarse). En este último caso, se establece una correspondencia con el grupo de los verbos pronominales. Nos podemos interrogar si esto siempre es así, ya que encontramos posibles contraejemplos como fallecer o desaparecer (El actor falleció ayer, Los documentos desaparecieron por la tarde), los cuales no toleran en principio el pronombre $s e^{7}$. Dichos verbos parecen codificar sendas relaciones de causatividad en las que, respectivamente, existe un motivo implícito en la muerte de una persona o en la que alguien provoca la desaparición de unos documentos. Partiendo de esta tesis, podemos afirmar que el pronombre que acompaña a los verbos télicos sirve para cancelar toda interpretación agentiva que surge naturalmente de ellos ${ }^{8}$.

Siguiendo a Vendler (1957), los eventos télicos como salir o enamorarse se encuadran dentro del grupo de los logros, los cuales son calificados erróneamente de 'puntuales'. Desde la teoría subeventiva, nosotros rechazamos el carácter puntual de dichos predicados, ya que la dinamicidad obliga a que todos sean durativos. Junto a los logros se encuentran otros predicados télicos llamados realizaciones (escribir una carta o ir al parque). Según se explica en Moreno Burgos (2014a: 121-125), la diferencia entre las realizaciones y los logros no es de naturaleza semántica, sino que surge pragmáticamente al asociar a un evento télico con un proceso. Así, por ejemplo, si una persona pide a otra que caliente la leche, el oyente interpretará que el resultado final depende de una acción realizada por él mismo, como puede ser la utilización de un microondas. Hace notar Verkuyl (1972) que la información aspectual de las realizaciones llega de modo composicional (esto es, mediante la adición de un complemento) y no por vía exclusivamente léxica como en eventos como enamorarse. Pues bien, una observación atenta de los datos nos lleva a poner en duda la cuestión de la composicionalidad.

En el caso de escribir se puede constatar que este verbo no siempre aparece acompañado por complementos como una carta. En este sentido, se puede decir tanto "ayer escribí toda la tarde" (sin más especificaciones) como "ayer escribí una carta”. Esto nos sitúa, sin embargo, frente a la posibilidad de distinguir entre dos tipos de predicados: un escribir léxicamente atélico y otro escribir léxicamente télico. Es necesario advertir que la presencia del complemento correspondiente supone indicar el soporte en el cual se realiza la acción designada, ya que lo que en realidad se escribe es un saludo o una queja. En consecuencia, esto implica la aparición de algo

7 Según el Diccionario de la Real Academia Española (DRAE), existe un uso pronominal de fallecer, el cual remite, no ya a la acción de morir, sino a otra en la cual algo se acaba. Con relación a desaparecer, se indica que en el español de Latinoamérica sí que se prevé la utilización del pronombre.

8 Existe la aparente excepción de comerse y beberse. Más adelante explicaremos a qué se debe. 
que antes no estaba ahí: 'se ha puesto algo sobre el papel'. En un estudio diacrónico, que no podemos acometer aquí, sería interesante realizar un análisis detallado del verbo latino scribere, el cual remite al significado de 'rayar'. Así aparece en el Diccionario de autoridades de la Real Academia: "Por antonomasia se entiende escribir con la pluma en papel, por ser lo más común y usual; pero también se escribe en las piedras y en los metales con el cincel o estilo de hierro: en los pergaminos, en lienzo bruñido, en las paredes y otras cosas".

En lo referente al verbo ir nos encontramos con un caso parecido, ya que este remite tanto a la atelicidad (ir por el parque) como a la telicidad (ir al parque). De nuevo aquí sería interesante enfrentar la hipótesis de la composicionalidad a la hipótesis léxica a partir de un estudio diacrónico. La cuestión es que, como ya se sabe, en el verbo ir español confluyen las formas de ire y vadere, lo cual podría verse reflejado en los diferentes valores aspectuales referidos. Al mismo tiempo, hay que recordar que en latín existe el denominado "acusativo de dirección", el cual aparece con o sin preposición. Así, Romam ire significaría “ir a Roma”, mientras que ad Romam ire sería más bien "ir, marchar hacia Roma”. Lo atractivo de esta observación es determinar si en ambos casos se trata de un argumento verbal.

Finalmente, es necesario indicar que existe una variante pronominal del verbo de movimiento, la cual aporta un matiz diferente: si ir al parque designa la meta, irse del parque constituye el lugar de origen. Esto permite, a su vez, establecer una relación de antonimia con el verbo quedarse, ya que mientras que este remite a la permanencia en un sitio (todavía), irse supone más bien dejar de estar en él ( ya no $)^{10}$. Este esquema es aplicable al doblete llevar/ llevarse, el cual añade un componente semántico adicional: 'desplazarse transportando algo'. No obstante, de la polisemia del pronombre se nos ocuparemos en el siguiente apartado más extensamente.

\subsection{El pronombre se}

Para realizar un análisis detallado del pronombre se, comenzaremos mostrando los siguientes enunciados:

(7) Miguel se cansó de que preguntaran tantas cosas y empezó a toser para que se marcharan (Ignacio Martínez de Pisón, 1994: La ternura del dragón, CREA).

(8) Movió la llave de contacto, se encendió el motor y tomamos el camino del sur hacia la península malaya (Manuel Leguineche, 1996: El camino más corto. Una trepidante vuelta al mundo en automóvil, CREA).

(9) Una joven culebra accedió a su laboratorio por los conductos del aire acondicionado y en una noche se comió tres pájaros (Miguel Delibes de Castro, 2001: Vida. La naturaleza en peligro, CREA).

$9 \quad$ Esto se puede poner en paralelismos con el alemán: Peter hat eine Vase mit Blumen bemalt (literalmente: 'Peter ha pintado un jarrón con flores') frente a Peter hat eine Blume gemalt ('Peter ha pintado una flor').

10 La presencia del pronombre en irse respondería, por tanto, a un fenómeno de analogía con quedarse. 
(10) Las persianas de material plástico son muy vulnerables: se rompen con cierta facilidad utilizando un cincel, un escoplo o un simple destornillador (Juan de Cusa, 1992: Seguridad en la vivienda, CREA).

(11) En el mundo se hablan alrededor de diez mil lenguas diferentes. Los lingüistas han llegado a emparentar la mayoría en familias (Jorge Urrutia, 1975: Sistemas de comunicación, CREA).

(12) La calle ha dejado de ser un espacio de sociabilidad y es habitada por los menos favorecidos". En su opinión, hoy se vive en la casa, el lugar de trabajo y la carretera (El País, 21/04/1997, CREA).

En el ejemplo (7) tenemos un evento télico (cansarse). Como ya hemos indicado, la presencia del pronombre es interpretado como que la acción ocurre de manera espontánea, mientras que la ausencia del mismo desbloquea dicha interpretación: si dijéramos que los invitados cansaron a Miguel con sus preguntas, obtendríamos una lectura causativa - que no agentiva. En el ejemplo (8) tenemos de nuevo un verbo télico. La comparación con el correspondiente verbo no-pronominal (Juan encendió el coche) permite, sin embargo, establecer una relación agentiva entre un sujeto personal y un nuevo estado de cosas ("el motor está encendido") que no surge con encenderse. Detengámonos aquí para entender la diferencia entre agentividad y causatividad:

\begin{tabular}{|l|l|c|c|c|}
\hline \multicolumn{2}{|l|}{} & TELICIDAD & AgentividAD & CAUsATIVIDAD \\
\hline 1 & Juan encendió la televisión & Sí & Sí & -- \\
\hline 2 & La televisión se encendió & Sí & No & No \\
\hline 3 & Juan se cansó de las preguntas & Sí & No & No \\
\hline 4 & César se murió de un infarto & Sí & No & No \\
\hline 5 & Las preguntas cansaron a Juan & Sí & No & Sí \\
\hline 6 & César murió de un infarto & Sí & No & Sí \\
\hline
\end{tabular}

Figura 2. Agentividad y causatividad según este trabajo.

Como observamos en el cuadro, tanto la agentividad como la causatividad son nociones relacionadas con los predicados télicos. La intencionalidad del primer nivel de representación permite identificar a un participante como agente, lo cual provoca que cualquier consideración sobre la causa sea irrelevante. En el resto de ejemplos se aprecia, sin embargo, que la falta de agentividad no siempre va pareja con la no-causatividad: esto solo se aplica a los niveles 2,3 y 4 , donde aparece el pronombre se; pero no a los niveles 5 y 6 . En efecto, en los dobletes como \{morir/ morirse\} de un infarto se trata de considerar si el infarto es la verdadera causa o más bien el inductor de algo que lleva a la muerte. Lo mismo ocurriría con cansar(se).

Siguiendo con el ejemplo (9) nos encontramos con un fenómeno más bien marginal, ya que atañe a un número reducido de predicados: el pronombre se aparece con verbos como comer, beber, leer o fumar, pero no con otros como escribir (se comió un bocadillo/ escribió una carta). En función de esto, en la bibliografía se aborda esta problemática desde diferentes enfoques. 
Así, observamos que De Miguel y Lagunilla (2000) adoptan la teoría subeventiva de Pustejovsky (1991), pero de una manera distinta a lo defendido aquí: según estas autoras, eventos como leer un libro (denominados por ellas transiciones) admiten el clítico porque constan de un subevento identificado con un logro. En nuestra opinión, sin embargo, los subeventos solo pueden ser estados. Por otro lado, García Fernández (2011: 57) considera que el pronombre se es opcional, postura con la que no estamos de acuerdo. Desde la argumentación de este autor, los dobletes dormir / dormirse no responden a las mismas motivaciones semánticas que comer / comerse; no obstante, nos cuesta seguir este razonamiento: en ambos casos la presencia del pronombre implica un mecanismo de telización evidente. Nosotros lo explicaremos partiendo del doblete acabar / acabarse, los cuales pueden parafrasearse como 'llevar a su fin' y 'llegar a su fin', respectivamente ${ }^{11}$. En primer lugar, el verbo no-pronominal remite a lecturas agentivas, como observamos en Juan ha acabado con las galletas; en segundo lugar, tendríamos lecturas no-agentivas como las que se registran en Se han acabado las galletas. En ambos casos, se debe interpretar una transición entre un estados de cosas en el que las galletas están ahí y otro en el que no están; esto es, desaparecen. Ahora bien, acabarse permite otra posibilidad, como se observa en la oración Juan se ha acabado las galletas. Lo particular de esta es que expresa una situación en principio atípica, ya que parece existir una compatibilidad entre el pronombre se y la agentividad. Nosotros lo explicaremos de la siguiente manera: más que llevar las galletas a un sitio (entendido metafóricamente como "el fin"), se trataría más bien de llevarse las galletas de algún sitio. Este paralelismo con el verbo llevarse permite explicar no solo la adición del correspondiente pronombre, sino también el hecho de que la agentividad percibida solo concierne al cambio de lugar, pero no a la finalización de las galletas. Teniendo en cuenta que existen diferentes maneras de acabarse las cosas, el uso del pronombre se extiende también a predicados del tipo comer: Juan se ha acabado las galletas> Juan se ha comido las galletas. El mecanismo telizador se aplica, por tanto, siguiendo el esquema de los verbos de desplazamiento locativo: ir y llevar son atélicos en el sentido de "caminar" y "transportar", respectivamente; lo mismo pasa con comer en ausencia del pronombre.

En el ejemplo (10) observamos un evento similar al que aparece en (8): se expresa la telicidad en un evento que ocurre de manera espontánea. Con una particularidad: el sujeto de la predicación aparece en plural y el verbo en presente, forma verbal de carácter imperfectivo. Esto provoca que se llegue a una lectura estativa de tipo genérica; esto es, en relación a una clase: la de las persianas (Mendikoetxea 1999). Este esquema permite en otros casos eliminar la mención explícita a un agente, como encontramos en Las camisas se planchan mal, lo cual estaría en el origen de oraciones de pasiva refleja como las de (11) o impersonales como las de (12). En un primer momento aparecen también en presente, pero posteriormente se combinarán con formas perfectivas (Ayer se cometieron dos atracos, A las tres se anunció los nombres de los ganadores). La diferencia entre pasivas e impersonales reside en que solo las primeras implican una reordenación de las funciones informativas, a partir de lo cual el objeto pasa a ser sujeto de la predicación.

11 El verbo acabar puede remitir a un valor no-agentivo en ejemplos como La película acabó a las diez. Ahí observamos que la preposición con no está exigida por el verbo y que el sujeto, por su parte, no es personal. La diferencia con respecto a La película se acabó a las diez reside en que en este último caso se considera que el punto de referencia temporal no se corresponde con lo esperado por el hablante. 
A continuación sintetizaremos en un cuadro lo dicho hasta ahora, con el fin de dejar constancia de los diferentes grados de interrelación que existen en los enunciados en los que aparece el pronombre se:

\section{EVENTOS}

\begin{tabular}{|l|c|}
\hline \multicolumn{2}{|c|}{ Verbos no-agentivos (télicos y pronominales) } \\
Morirse, cansarse, romperse \\
\hline Causatividad \\
Morir, cansar
\end{tabular}

\section{ESTADOS}

\begin{tabular}{|c|c|}
\hline \multicolumn{2}{|c|}{ Genericidad } \\
\hline Las camisas se planchan mal \\
\hline En España se hablan cuatro lenguas & Impersonal \\
Aquí se vive bien \\
\hline
\end{tabular}

Figura 3. Diferentes valores de se según nuestra propuesta.

Por último mostraremos que existen otros casos en los que el pronombre se constituye un alomorfo del pronombre le, razón por la cual lo desvincularemos de la telicidad. Se trata de relaciones de posesión inalienable y recíprocas ${ }^{12}$. Observemos los siguientes ejemplos:

(13) Vino el niño con una jofaina y Emeterio se lavó las manos y la cara con mucho esmero, restregándose uñas y orejas (Germán Sánchez Espeso, 1985: En las alas de las mariposas, CREA).

(14) En su juventud todavía se escribían cartas de amor. Querido Daniel, Daniel, mi vida. Por lo que más quieras, Daniel, dime si te acuerdas de mí, dime algo (Carmen Martín Gaite, 1994: Nubosidad variable, CREA).

En la oración (13) se expresa que las manos son las de propio sujeto de la predicación. De hecho, en lenguas como el inglés, donde no aparece ningún pronombre, se hace necesaria la presencia de un posesivo (He washed his hands). Según el DRAE, el origen de este pronombre se sitúa en sui, el cual se corresponde con el genitivo y bien es sabido que dicha información se expresa en las lenguas romances a través de complementos introducidos por la preposición de ('lava la cara de sí mismo'); en una etapa siguiente, este se reinterpretará como un dativo ('se lava la cara a sí mismo'). En este sentido, opinamos que el pronombre adopta la misma función que en oraciones como Le ha lavado las manos a su hijo, donde interviene un tercer participante ${ }^{13}$.

12 Renunciamos voluntariamente al término reflexivo para evitar la confusión entre los diferentes predicados pronominales que este término engloba. Piénsese, por ejemplo, en el verbo llamarse, que ha lexicalizado la expresión de la estatividad.

13 En alemán no existe esta ambigüedad en relación a los pronombres de primera y segunda persona, ya que se marca el caso dativo de manera inequívoca: ich wasche mir die Hände ('Me lavo las manos'). 
Las oraciones recíprocas como las de (14) conceptualizan igualmente una relación semántica en la que aparece involucrada una tercera entidad. Así, llegamos a la conclusión de que Daniel le escribía cartas a una persona y esa persona a su vez le escribía a Daniel. Esto es, el pronombre se adopta de nuevo aquí la función del caso dativo (se escribían cartas a ellos mismos). Desde estas bases podemos explicar que el fenómeno de disimilación que se da al combinar pronombres de tercera persona no solo responde a razones fonéticas, sino que constituyen un caso de analogía con respecto a los casos anteriores:

(15) En el estante indicado vio un devocionario viejo, un rosario de cuentas blancas y una pulsera de nardos naturales [...]. También había un mazo de cartas; lo cogió y se lo dio a la vidente, que había abierto los párpados (Eduardo Mendoza, 1993: La ciudad de los prodigios, CREA).

En efecto, las cuestiones relacionadas con la eufonía no suponen una argumentación definitiva, sino que es necesario tener en cuenta el componente semántico subyacente.

\section{Agentividad y estados}

\subsection{Exposición de los datos}

Una vez definido lo que entendemos por agentividad, pasaremos a analizar secuencias como las que hemos presentado en la introducción bajo los números (1) y (2). Nuestra hipótesis será considerar que dicha lectura no llega por vía aspectual, sino modal. Sin embargo, antes de detallar estas ideas, presentaremos cuál es el posicionamiento de algunos autores en relación al fenómeno.

En primer lugar, debemos citar a Davidson (1967), quien propugna la existencia de un argumento eventivo en los predicados dinámicos. Según esta hipótesis, la dinamicidad debe ser entendida como un papel temático paralelo a los ya aludidos de agente, tema, causa, etc. ${ }^{14}$ En esta línea de análisis, autores como Kratzer (1995) defienden que dicho argumento eventivo también es aplicable a ciertos estados, que en español se correspondería con aquellos en los que aparece estar. Esta última afirmación viene a ser controvertida, dado que como indican Marín (2013) o Maienborn (2005), ello impide trazar una frontera clara entre estados y eventos. Sin embargo, el primer autor contempla una excepción en la que sí sería relevante la teoría aludida: el grupo de los que él denomina adjetivos evaluativos de comportamiento, tales como amable, cruel o honesto. A nosotros nos parece igualmente inadecuada dicha especificación, ya que se produce un salto conceptual del dominio verbal al adjetival, no quedando claro a qué plano queda ligado el argumento davidsoniano. Parece evidente que la agentividad está contenida en la semántica de los adjetivos, pero no que esta tenga una relación directa con los estados ${ }^{15}$.

14 Esta postura nos parece de entrada problemática, ya que la estructura argumental debería ser dependiente de la dinamicidad, más bien que encontrarse al mismo nivel.

15 Compárese con la lectura agentiva de determinados sustantivos (descubridor, traductor, fundador), la cual llega por procedimientos morfológicos (Cano Cambronero 2013). 
Esto es, observamos que la semántica adjetival es una condición necesaria, pero no suficiente, ya que la agentividad solo es palpable en el caso de que las piezas léxicas se combinen con determinadas formas verbales como el pretérito indefinido o el imperativo, según lo visto en la introducción.

Cuartero Otal y Horno Chéliz (2011: 230) reflexionan igualmente sobre la relación entre estados y agentividad a partir de ejemplos como Sé juicioso o Procuraré ser juicioso. Llegan a la conclusión de que, dado que no existe incompatibilidad entre ambas nociones, es pertinente tener en cuenta el argumento davidsoniano. Y añaden que "al focalizar una fase estativa, el predicado se está proyectando con su argumento eventivo ya saturado con la información de estado" (Cuartero Otal y Horno Chéliz 2011: 244). Como se puede observar, esta explicación tampoco permite diferenciar claramente entre eventos y estados, quizá porque los autores parten de una idea preconcebida sobre la que hemos advertido en la introducción: la supuesta duratividad de los estados. Eso les lleva a considerar que los estados son atélicos; sin embargo, la atelicidad es una característica únicamente atribuible a los predicados dinámicos $\mathrm{y}$, como sabemos, los estados no presentan evolución temporal. Por otro lado, opinan que los estados pueden expresar gradación, lo cual pretenden demostrar mediante oraciones como Max empezó a sentirse enfermó. Esto induciría a pensar que los estados son predicados complejos y no nucleares como nosotros defendemos.

Desde la teoría subeventiva de Pustejovsky (1991), profundizada por Moreno Cabrera (2003), se llega a un análisis coherente del fenómeno de la aspectualidad, el cual implica dos niveles de representación en torno a un criterio binario de presencia o ausencia de dinamicidad. Los autores que defienden la divisibilidad de los estados se encuentran frente a un problema teórico adicional que les obliga a definir la naturaleza de sus partes integrantes. Y ahí es donde las hipótesis divergen: o bien no se hacen puntualizaciones al respecto o bien nos sitúan en dificultades ontológicas de difícil resolución. Este es el caso de Cunha (2007: 86), quien habla de estados faseables a propósito de ejemplos como Maria, sê cuidadosa! (‘¡María, sé cuidadosa!’): si tanto estados como eventos estuvieran formados por fases, la teoría nunca podría predecir cuándo nos encontramos ante unos $\mathrm{u}$ otros.

Arche (2011: 104) habla de una interpretación eventiva implícita con respecto a enunciados como Juan fue cruel con Pedro, el cual se puede explicitar mediante determinadas cláusulas (al invitar a su exnovia a la fiesta). La autora lo justifica diciendo que "el adjetivo se predica del evento"; sin embargo, desde esta perspectiva estaríamos eliminando la agentividad atribuida al estado ser cruel. Esto es, la semántica del predicado estativo contraería una relación de interdependencia con respecto a una cláusula subordinada elíptica, algo difícil de sostener.

En nuestra opinión, todos estos estudios intentan explicar las interpretaciones agentivas de los estados como si estos fueran eventos. Sin embargo, dado que los estados no son dinámicos, dicha agentividad debe llegar por otros cauces, que nosotros identificamos con la modalidad. Este concepto, definido por Lyons (1977) está relacionado con el contenido de verdad de las proposiciones en el momento del habla ${ }^{16}$ y puede ser expresado de diferentes maneras: mediante el subjuntivo (No creo que sea español), mediante usos dislocados del futuro (No ha venido, estará

16 Es lo que se conoce como modalidad epistémica; también se habla de modalidad deóntica, la cual remite a valores de obligación. Nosotros consideramos que esta última solo afecta a los eventos y, por tanto, no será pertinente en nuestro análisis. 
enfermo) o mediante determinadas perífrasis (Tiene que tener unos 30 años). Según hemos visto, la combinación de determinados estados con el imperativo (Sé amable) o con formas perfectivas (Fuiste muy cruel) da lugar a esa misma lectura, dado que se pone en duda que el interlocutor sea amable o cruel respectivamente. Desde el punto de vista interpretativo se considera que existe un comportamiento de fondo, en el cual está obligatoriamente implicada la semántica de los adjetivos ${ }^{17}$. Es decir, se interpreta como si fuera un cambio de estado de no-ser-amable a seramable en el caso del imperativo. En lo relativo a las formas perfectivas, se trata más bien de la dirección contraria: si alguien era cruel en el pasado es posible que siga siéndolo en el presente; sin embargo, si alguien fue cruel en el pasado, ya no lo es en el momento del habla. En otras palabras, la perfectividad de los eventos conceptualiza un esquema que puede ser explicitado mediante ya no. A continuación explicaremos brevemente el alcance de esta afirmación.

Partiendo de los trabajos de Muller (1975) y Garrido (1992), consideramos que el adverbio ya permite dejar al descubierto diferentes fases en relación a un evento. Así, si pensamos en la oración Juan ya ha llegado, observamos que se focaliza un estado de cosas inmediatamente posterior a la llegada de Juan; esto es, “Juan está aquí”. Pero al mismo tiempo se introduce una presuposición (“Juan no estaba aquí antes”) y una expectativa (“Juan seguirá aquí después”). Esta tesis encaja a la perfección con la teoría aspectual de Klein (1992), quien establece una relación entre el denominado tiempo de la situación (en este caso, el evento llegar) y el tiempo del foco (la fase en relación a dicho evento). La negación de ya se expresa de la siguiente manera: Juan todavía no ha llegado; esto es, existe una fase negada predicada (“Juan no está aquí”) y dos no-predicadas: una presuposición (“Juan no estaba aquí antes”) y una expectativa ("Juan estará aquí después”). Esto es lo que denomina Klein (1992) aspecto Perfecto, el cual no debe ser confundido con el Perfectivo o Aoristo ${ }^{18}$. Ahora bien, en complemento a estas teorías, nosotros opinamos que la semántica del adverbio todavía (y su contrapartida ya no) está más bien relacionada con la veracidad de las proposiciones. Esto se traduce en lo siguiente: mientras que el Aoristo de los eventos implica que el tiempo del foco coincide con la totalidad del tiempo de la situación, el Aoristo de los estados debe ser puesto en relación con la modalidad. De este modo, partiendo de un enunciado como Juan era profesor en su juventud podemos llegar a dos conclusiones lógicas: que Juan ya no es profesor, o que Juan todavía lo es. En el primero de los casos se llega, por tanto, a una lectura perfectiva: Juan fue profesor ${ }^{19}$.

Una vez explicados estos casos, en el próximo apartado aportaremos más pruebas de que la agentividad en los estados está directamente relacionada con la modalidad. Se trata de contextos que incluyen la perífrasis <estar + gerundio>, en los que aparece el pronombre clítico se o que remiten a la habitualidad.

17 Lógicamente, se impone que el sujeto sea personal (cf. El cambio fue muy brusco).

18 Klein (1992) emplea por el primero de los términos; nosotros, siguiendo a Bertinetto (1986) preferimos el segundo, para evitar así confusiones terminológicas.

19 No nos detendremos más en ello. Para más detalles, consúltese Moreno Burgos (2014b). 


\subsection{Implicaciones}

En este apartado profundizaremos en nuestra tesis de que la agentividad vinculada a ciertos estados no surge a partir del aspecto gramatical. Desde esta perspectiva analizaremos los siguientes ejemplos:

(16) Al entrar ella en el coche comprendió que estaba siendo muy brusco con ella y atrayéndola le besó intensamente en los labios (José María Guelbenzu, 1989: El río de la luna, CREA).

(17) Siempre era amable con él, y lo seguía llamando ahijado como en los viejos tiempos (Arturo Pérez-Reverte, 2002: La Reina del Sur, CREA).

(18) Descendió una escalera, sintiendo un [sic] mano robusta que le sujetaba por un brazo, y al final se estuvo quieto, esperando algo (Juan Eduardo Zúñiga, 1990: Largo noviembre de Madrid, CREA).

El primero de los enunciados contiene una perífrasis sobre la cual no existe a día de hoy una definición unánime en la bibliografía. La mayoría de los autores interpreta que <estar + gerundio> posee un significado aspectual; sin embargo, siguiendo a Moreno Burgos (2016), defenderemos que esto no es aplicable al ejemplo (16), ya que ahí tenemos una estructura homónima de tipo modal. En efecto, aquí no se trata de focalizar la parte interna de un evento que se encuentra en desarrollo en el momento del habla, sino de poner en duda que al sujeto de la predicación se le pueda asignar una propiedad determinada (ser brusco). Obsérvese de nuevo que la lectura agentiva no siempre aparece expresada: en enunciado como El artista está siendo víctima de una extorsión se interpreta modalmente, pero no en virtud de un comportamiento.

El ejemplo que aparece en (17) constituye una prueba de que únicamente determinados adjetivos permiten la expresión de la habitualidad, los cuales coinciden con los que presentan la agentividad señalada en este trabajo. Esto explica, por tanto, la anomalía de enunciados como Siempre soy profesor ${ }^{20}$. El componente modal necesario para llegar a dicha interpretación es la propia lectura habitual: en Moreno Burgos (2014a: 307-315) se pone en relación con la lectura epistémica que se registra en oraciones como El tren salía a las ocho y media, ¿no?, mediante las cuales el hablante expresa sus reservas sobre la veracidad de un 'presente pro futuro'. No nos detendremos aquí en especificar más detalles; simplemente expondremos un argumento de peso: mientras que la expresión del tiempo gramatical (p.e. en las oraciones Juan llegó a las seis o Juan estaba estudiando a las seis) implica el anclaje de una situación (estado o evento) en un punto de referencia, el concepto de 'modalidad' excluye precisamente dicho anclaje. Esto es, basándonos en la teoría de Reichenbach (1947), no contemplamos que pueda existir una localización múltiple de un evento como ir al cine en el eje de la temporalidad (Juan iba todos los jueves al cine); sino que, como en el ejemplo (16), se trata de evaluar la veracidad del enunciado en el momento del habla.

En relación a ejemplos como el de (18) encontramos los estudios de Morimoto (2008: 596) y García Fernández (2011: 69). Según ambos autores, la presencia del pronombre clítico es debida

20 Nótese, sin embargo, que la habitualidad no está descartada en el caso del verbo estar, aun en el caso de que no haya una lectura agentiva. Si partimos de un enunciado como Siempre está cansado, observamos que se considera de fondo la repetición de un proceso que lleva al estado de cosas descrito. 
a la naturaleza controladora del sujeto, afirmación con la que no estamos de acuerdo. Nuestra argumentación es la siguiente: según lo defendido en este artículo, la agentividad que se aprecia en la oración (18) no viene dada por vía aspectual, sino modal; es decir, por el uso de una forma perfectiva. Una prueba de ello es que podemos suprimir el pronombre clítico sin que eso repercuta en la lectura agentiva: al final estuvo quieto, esperando algo. El hecho de que dicho pronombre pueda aparecer lo relacionaremos con la proximidad semántica que existe con el predicado quedarse. Lo interesante de este verbo es que ha sufrido un proceso de gramaticalización mediante el cual ha perdido sus propiedades dinámicas y se ha transformado en estático. Esto es, originalmente estaba relacionado con la falta de movimiento y venía a ser un sinónimo del verbo pararse, como apreciamos en predicaciones ya en desuso como estar quedo ('estar quieto'). En una etapa posterior, se convierte en idóneo para expresar relaciones locativas similares a las que introducen los estados identificados con estar (quedarse en casa), constituyéndose además como el antónimo de irse - según hemos avanzado anteriormente. No obstante, en consonancia con la semántica de estar, el verbo quedarse amplía su radio de aplicación hasta contextos que no son forzosamente locativos: \{estar/ quedarse\} callado.

Ahora bien: en relación a (18) nos queda un problema teórico que solucionar. Si la lectura agentiva surge a través de la modalidad y el pronombre se no es en este caso obligatorio, ¿por qué no ocurre lo mismo en el caso del imperativo? En efecto, observamos que un enunciado como ¡Está callado! resulta anómalo entendido como una orden, en oposición a ¡Estate callado! Nosotros consideramos que se trata de un recurso de desambiguación, con el fin de evitar las confusiones con la tercera persona de singular del presente de indicativo. La prueba es que la negación del imperativo no impone dicho requisito: No estés tan callado.

\section{Conclusiones}

La conclusión principal a la que se llega en este trabajo es que la agentividad es una lectura derivada que llega por dos vías: la aspectual y la modal. La primera de ellas se debe a que se parte de las propiedades dinámicas de los eventos télicos y se infiere un significado intencional. La segunda surge al vincular a los estados con su contenido de verdad, la cual llega por diversas vías: las formas perfectivas, el imperativo, la perífrasis <estar + gerundio > o la habitualidad. Sin embargo, además de los respectivos componentes semánticos relacionados con la telicidad y la modalidad, se impone un requisito de base: la lectura agentiva solo puede ser desempeñada por un sujeto personal; esto es, un ser humano. En el caso de que no se establezca un vínculo directo entre la acción y el resultado (ya sea por la falta de intencionalidad del sujeto o porque directamente no se trate de una persona), lo más adecuado es hablar de causa y no de agente. Por otro lado, hemos observado que la semántica del pronombre se debe ser descrita en una primera instancia como un mecanismo para despojar a los verbos télicos de su lectura de agentividad. A partir de ahí observamos un desarrollo polisémico de dicho pronombre que hemos resumido en la figura 3. A ello se le suma la confluencia de otro pronombre homónimo que remite al caso dativo.

Al hilo de todo esto surgen interesantes líneas de investigación que podemos enumerar de la siguiente manera: 1) Si únicamente los eventos télicos poseen un agente, habría que reconsiderar el papel temático que se le atribuye al sujeto de los predicados atélicos. 2) El uso del 
pronombre se podría responder en ocasiones a usos estilísticos, como observamos en el doblete morir/ morirse. Esto es, según lo expuesto aquí, la alternativa menos marcada sería la pronominal; sin embargo, en el lenguaje periodístico prevalece la no-pronominal, posiblemente porque los comunicadores de una noticia se ven obligados a presentar cuáles son las causas de los sucesos. 3) La ausencia o presencia de dicho pronombre no es uniforme en todas las variedades geográficas del español. Anteriormente hemos aludido al verbo desaparecer(se), con lo cual sería interesante determinar los factores semánticos que motivan esta disparidad y si ello responde a desarrollos evolutivos más o menos avanzados.

En definitiva, al considerar que la agentividad es un fenómeno derivado, estamos defendiendo que se trata de un traspaso semántico del dominio verbal al dominio nominal representado por el sujeto. Por esta razón sería inadecuado hablar de 'verbos agentivos'.

\section{Referencias bibliográficas}

Arche, $\mathrm{M}^{\mathrm{a}} \mathrm{J}$. (2011). Las oraciones copulativas agentivas. In $\mathrm{M}^{\mathrm{a}} \mathrm{V}$. Escandell et alii (Eds.), 60 problemas de gramática (pp. 99-105). Madrid: Akal.

Beck, G. (1987). Verb-Satz-Zeit. Zur temporalen Struktur der Verben im Französischen. Tübingen: Max Niemeyer.

Bertinetto, P. M. (1986). Tempo, aspetto e azione nel verbo italiano. Firenze: Academia della Crusca.

Cano Cambronero, $\mathrm{M}^{\mathrm{a}} \mathrm{A}$. (2013). Las derivaciones en -nte y -dor: Estructura argumental y complejidad sintáctica en una morfología neoconstruccionista. Universidad Autónoma de Madrid: tesis doctoral. <https:// repositorio.uam.es/handle/10486/661875>.

Carlson, G. N. (1978). Reference to kinds in English. Bloomington: Indiana University Linguistics Club.

Cuartero Otal, J. et alii (Eds.). (2011). Estudios sobre perifrasis y aspecto. München: Peniope.

Cuartero Otal, J., \& Horno Chéliz, Ma (2011). Estados, estatividad y perífrasis. In J. Cuartero Otal et alii (Eds.), (pp. 225-248). München: Peniope.

Cunha, L. F. (2007). Semântica das predicações estativas. Para uma caracterização aspectual dos estados. München: Lincoln Europa.

Davidson, D. (1967). The logical form of action sentences. In N. Rescher (Ed.), The logic of decision and action (pp. 81-95). Pittsburgh: University of Pittsburgh.

De Miguel, E., \& Fernández Lagunilla, M. (2000). El operador aspectual se. Revista de lingüística española, 30(1), 13-45.

Dik, S. C. (1997). The theory of functional grammar. Part 1. The structure of the clause. Berlin/ New York: Mouton de Gruyter.

Dowty, D. (1979). Word meaning and Montague grammar. The semantics of verbs and times in generative semantics and in Montague's PTQ. Dordrecht-Boston-London: Kluwer Academic Publishers.

García Fernández, L. (2011). Algunas observaciones sobre se aspectual. In J. Cuartero Otal et alii (Eds.), (pp. 43-71). München: Peniope.

Garrido, J. (1992). Expectations in Spanish and German adverbs of change. Folia Linguistica, 26, 357-402.

González Rodríguez, R. (2011). Eventos negativos y perífrasis verbales. In J. Cuartero Otal et alii (Eds.), (pp. 99-119). München: Peniope.

Kratzer, A. (1995). Stage-level and Individual-Level predicates. In G. N. Carlson, \& F. J. Pelletier (Eds.), The generic book (pp. 125-175). Chicago-London: University of Chicago. 
Lakoff, G. (1970). Irregularity in syntax. New York: Holt, Rinehart and Winston.

Langacker, R. (1987). Foundations on cognitive grammar: Theoretical Prerequisites (vol. I). Standford: Standford University Press.

Lyons, J. (1977). Semantics. London: Cambridge University Press.

Maienborn, C. (2005). A discourse-based account of Spanish ser / estar. Linguistics, 43(1), 155-180.

Marín, R. (2013). La stativité dans tous ses états. Université Paris 8. Tesis de habilitación. <https://www. researchgate.net/publication/260026245_La_stativite_dans_tous_ses_etats $>$.

Mendikoetxea, A. (1999). Construcciones con se: Medias, pasivas e impersonales. In I. Bosque Muñoz, \& V. Demonte Barreto (Dirs.), Gramática descriptiva de la lengua española (pp. 1631-1722). Madrid: Espasa Calpe.

Moreno Burgos, J. (2014a). Estatividad y aspecto gramatical. Universität Regensburg. Tesis doctoral. <http:// epub.uni-regensburg.de/30467/>.

—. (2014b). Estados y perfectividad en español. Signos lingüísticos, 19, 8-37. <http://dcsh.izt.uam. $\mathrm{mx} /$ publicaciones/filosofia/index.php/SLING/article/view/1096>.

- (2015). El pretérito perfecto compuesto en el ámbito hispánico. Anuario de letras, 3(1), 87-130. $<$ https://revistas-filologicas.unam.mx/anuario-letras/index.php/al/issue/view/4/showToc $>$.

- (2016). La perífrasis <estar+gerundio> no es siempre aspectual. Cuadernos de Investigación Filológica, 42, 145-172. <https://publicaciones.unirioja.es/ojs/index.php/cif/issue/view/191>.

Moreno Cabrera, J. C. (2003). Semántica y gramática. Sucesos, papeles semánticos y relaciones sintácticas. Madrid: Antonio Machado libros.

Morimoto, Y. (2008). Me estuve quieto: el concepto de estado y el llamado se aspectual. In I. Olza Moreno et alii (Eds.), Actas del XXXVII Simposio Internacional de la Sociedad Española de Lingüística (SEL) (pp. 591-599). Pamplona: Servicio de Publicaciones de la Universidad de Navarra.

Muller, C. (1975). Remarques syntactico-sémantiques sur certains adverbes de temps. Le français modern, 43(1), 12-38.

Pustejovsky, J. (1991). The syntax of event structure. In B. Levin, \& S. Pinker (Eds.). Lexical and conceptual structure (pp. 47-81). Oxford: Blackwell.

[CREA]: Real Academia Española. Corpus de referencia del español actual. <http://www.rae.es>

—. Diccionario de autoridades. <http://web.frl.es/DA.html>.

- (2014). Diccionario de la lengua española. <http://www.rae.es/drae>.

Real Academia Española, Asociación de Academias de la Lengua Española (2010). Nueva gramática de la lengua española. Manual. Madrid: Espasa Libros.

Reichenbach, H. (1947). Elements of symbolic logic. New York/ London: The Free Press / Collier-Macmillan.

Tesnière, L. (1959). Éléments de syntaxe structurale. Paris: Klincksieck.

Vendler, Z. (1957). Verbs and times. Philosophical Review, 56, 143-160. [Reproducido en Vendler, Z. (1967). Lingusitics in Philosophy (pp. 97-121). Itaca-New York: Cornwell University Press.]

Verkuyl, H. (1972). On the compositional nature of the aspects. Dordrecht: Reidel. 